\title{
The Effects of Iron Supplementation on the Growth Rate and Antioxidant Activity of Trichomonas vaginalis
}

\author{
SM Razavi', E Foroudi ${ }^{1}$, E Rakhshandehroo ${ }^{1}$, F Nejati ${ }^{1}$ and S Nazifi ${ }^{*}$
}

${ }^{1}$ Department of Pathobiology, School of Veterinary Medicine, Shiraz University, Shiraz, Iran

${ }^{2}$ Department of Clinical Studies, School of Veterinary Medicine, Shiraz University, Shiraz, Iran

\begin{abstract}
Trichomonas vaginalis is a common protozoan parasite found in females and males worldwide. The parasite causes mild to severe inflammations in the urogenital tract. This study was conducted to evaluate the effect of iron excess on the growth rate and the activity of antioxidant enzymes in $T$. vaginalis. The parasite was cultured in Diamond's Trypticase Yeast Maltose (TYM) media with and without iron supplementation and assessed at 2, 3, 5, 7, 9, 11, 13 and 15 days post inoculation (dpi). The results showed significant higher numbers of the parasite in medium with iron supplementation. Furthermore, our findings revealed higher activities of antioxidant enzymes (SOD, GPX and CAT) in parasites cultured with iron supplementation. In conclusion, the present experiment showed that iron increased not only the multiplication rate but also the antioxidant activities of $T$. vaginalis. It seems that iron could protect $T$. vaginalis from toxic oxygen metabolites during tissue invasion and helps the parasite to maintain its pathogenicity for the host.
\end{abstract}

Keywords: Trichomonas vaginalis; Iron; Growth; Antioxidant enzymes

\section{Introduction}

Trichomonas vaginalis, a flagellated protozoan parasite, causes a prevalent sexually transmitted disease worldwide. The parasite mainly affects the urogenital tract of both men and women. In women, symptoms are heavier which range from mild to severe inflammation with a frothy malodorous discharge and severe irritation [1]. The parasite can invade the squamous epithelium and associates with vaginitis, low birth weights and many perinatal complications [2].

T. vaginalis lacks the ability to synthesize many of the macromolecules de novo. Therefore, the uptake of nutrients is from the vaginal secretions or through the host and/or bacterial cells [3]. This implicates that $T$. vaginalis is required to include many of the essential macromolecules in culture media. Among those molecules, vitamins and minerals such as iron seem very important for the survival of the parasite [4].

Iron is required to maintain maximal levels of ferredoxin and pyruvate-ferredoxinoxido reductase activity [5]. Also, it appears that resistance to complement-mediated lysis is dependent upon a high concentration of iron [6]. Iron has been demonstrated to upregulate the expression of cysteine proteinases, which have been found to degrade the $\mathrm{C} 3$ portion of complement on the surface of the organism which allows the organism to evade complement-mediated damage [6]. In addition, the pathogenesis of T. vaginalis is differentially modulated by iron [7]. Considering the role of iron for the pathogenesis Trichomonas in the host, it can be assumed that this element could have a significant effect on the growth rate of the parasite.

Free radicals are very reactive chemical substances which can cause oxidative damages to the living organisms. Under normal physiological conditions, there is a critical balance in the generation of free radicals and antioxidant defense systems against free radical injury $[8,9]$. The imbalance between free radical production and antioxidant defense creates a condition known as oxidative stress. Clinical observations and experimental evidence suggest that oxidative stress plays a dominant role in the host's defense against parasitic infections [10].

The function of antioxidant enzymes has to some extent been studied in ruminant hosts infected with the blood protozoan parasites [11-13].
The presence of superoxide dismutase (SOD) and NADH oxidases was reported in T. vaginalis $[14,15]$. However, the mechanisms of cellular protection against adverse effects of oxygen metabolites have not been investigated well in trichomonads. Therefore, this study was conducted in order to investigate the status of the iron supplementation on the growth rate and the activity of major antioxidant enzymes (glutathione peroxidase (GPX), superoxide dismutase (SOD) and catalase (CAT) in T. vaginalis.

\section{Materials and Methods}

\section{T. vaginalis cultivation}

A T. vaginalis isolate, kindly obtained from Shiraz University of Medical Sciences, Shiraz, Iran was routinely cultured in Diamond's TYM medium [16], composed of $150 \mathrm{mg}$ of Tryptone, $12 \mathrm{~g}$ of yeast extract, $5.5 \mathrm{~g}$ of glucose, $2.5 \mathrm{~g}$ of sodium chloride, $0.5 \mathrm{~g}$ of L-cysteine, 0.5 $\mathrm{g}$ of sodium thioglycolate, $80 \mathrm{mg}$ of Gentamicin, $2 \mathrm{mg}$ of Amphotericin $\mathrm{B}$, Penicillin $\mathrm{G}\left(1 \times 10^{6} \mathrm{SI}\right), 120 \mathrm{ml}$ of horse serum and $0.75 \mathrm{~g}$ of agar. The medium was constituted per $1000 \mathrm{ml}$ of distilled water and its $\mathrm{pH}$ was set at 6.2 .

The parasites were recultured in TYM media with and without iron supplementation and kept at $37^{\circ} \mathrm{C}$. Each group consisted of 48 microtubes containing $1.2 \mathrm{ml}$ culture media and $10 \mu \mathrm{l}$ media containing about 3000 T. vaginalis organism. In Iron-supplemented group, ferrous sulfate was also added to adjust a final Fe concentration on $400 \mu \mathrm{M}$ [ [ $]$ ]. Six microtubes from each group were removed and followed at 2, 3, 5,

${ }^{*}$ Corresponding author: Dr. Saeed Nazifi, Professor of Veterinary Clinical Pathology, Department of Clinical Studies, School of Veterinary Medicine Shiraz University, Shiraz, P.O. Box: 1731- 71345, Iran, Tel: +98-711-2286940; Fax: +98-711-2286950; E-mail: nazifi@shirazu.ac.ir

Received July 19, 2017; Accepted July 30, 2017; Published July 31, 2017

Citation: Razavi SM, Foroudi E, Rakhshandehroo E, Nejati F, Nazifi S (2017) The Effects of Iron Supplementation on the Growth Rate and Antioxidant Activity of Trichomonas vaginalis. J Vet Sci Technol 8: 463 . doi: 10.4172/2157-7579.1000463

Copyright: (C 2017 Razavi SM, et al. This is an open-access article distributed under the terms of the Creative Commons Attribution License, which permits unrestricted use, distribution, and reproduction in any medium, provided the original author and source are credited. 
$7,9,11,13$ and 15 days post inoculation (dpi). The cultured specimens were centrifuged at $3000 \mathrm{~g}$ for $3 \mathrm{~min}$; the pellet was resuspended in $1 \mu \mathrm{L}$ of sterile distilled water, shaken thoroughly and examined by a Neubauer cell chamber to calculate the parasite numbers per $\mathrm{ml}$. Finally, the contents of each micro tube were kept at $-20^{\circ} \mathrm{C}$ until the biochemical assays were performed.

\section{Biochemical assays}

Glutathione peroxidase (GPx) activity: The activity of GPx was evaluated with GPx detection kit (Ransel kit produced by Randox Co., UK) according to the manufacturer's instructions. GPx catalyze the oxidation of glutathione $(\mathrm{GSH})$ by cumene hydroperoxide. In the presence of glutathione reductase (GR) and NADPH, the oxidized glutathione (GSSG) is immediately converted to the reduced form with a concomitant oxidation of NADPH to NADP+. The decrease in absorbance at $340 \mathrm{~nm}$ against blank was measured spectrophotometrically. One unit (U) of GPx activity was defined as the amount of enzyme that converts $1 \mu \mathrm{mol}$ of NADPH to NADP+ per minute. The GPx activity was expressed as unit per mg of protein (U/ mg protein).

Superoxide dismutase (SOD) activity: SOD detection kit (Ransod", Randox Co., UK) was used to evaluate total SOD activity. This method employs xanthine and xanthine oxidase (XOD) to generate superoxide radicals which react with 2-(4-iodophenyl)-3-(4-nitrophenol)-5phenyltetrazolium chloride (INT) to form a red formazan dye. The SOD activity is then measured by the degree of inhibition of this reaction. One unit of SOD is that which causes 50\% inhibition of the rate of reduction of INT under the conditions of the assay. SOD levels were recorded at $505 \mathrm{~nm}$ and through a standard curve, and expressed as unit per $\mathrm{mg}$ of protein (U/mg protein).

Catalase (CAT) activity: Tissue catalase activity was assayed spectrophotometrically by monitoring the decomposition of $\mathrm{H}_{2} \mathrm{O}$ using the procedure of Aebi [17]. Briefly, $0.5 \mathrm{ml}$ of $30 \mathrm{mmol} / 1 \mathrm{H}_{2} \mathrm{O}$ solution in $50 \mathrm{mmol} / \mathrm{l}$ phosphate buffer $(\mathrm{pH}=7.0)$ and $1 \mathrm{ml}$ of $1: 10$ diluted tissue supernatant was added and the consumption of $\mathrm{H}_{2} \mathrm{O}$ was followed spectrophotometrically at $240 \mathrm{~nm}$ for $2 \mathrm{~min}$ at $25^{\circ} \mathrm{C}$. The molar extinction coefficient was $43.6 \mathrm{l} / \mathrm{mol}$ per $\mathrm{cm}$ for $\mathrm{H}_{2} \mathrm{O}$. Catalase activity was expressed as the unit that is defined as $\mu \mathrm{mol} \mathrm{H}_{2} \mathrm{O}$ consumed/min per $\mathrm{mg}$ of protein. In order to achieve the average activity of each enzyme in the whole parasite population, the values measured for each case were divided into the parasite number.

\section{Statistical analysis}

Student's $t$ test was used to compare the effects of iron supplementation on the evaluated parameters. All data were analyzed using the Statistical Package for Social Sciences (SPSS, 16.0) and the significance was set at $\mathrm{P}<0.05$.

\section{Results}

The pattern of alterations in the parasite number of both culture media is presented in Figure 1. Our data showed that although the parasites number was increased in both media from the start of the study toward the end, it was overall higher in iron-rich medium. Significant elevations of parasite number were seen at 2, 5, 7 and 15 days post inoculation (dpi) in iron-rich medium compared to usual Diamond's TYM medium. The alterations in the activity of antioxidant enzymes in different days are presented in Figure 2. The results revealed that the activity of GPx was significantly higher in parasites cultured in iron-rich medium. These differences were recorded at 2, 3, 5, 7, 11, 13

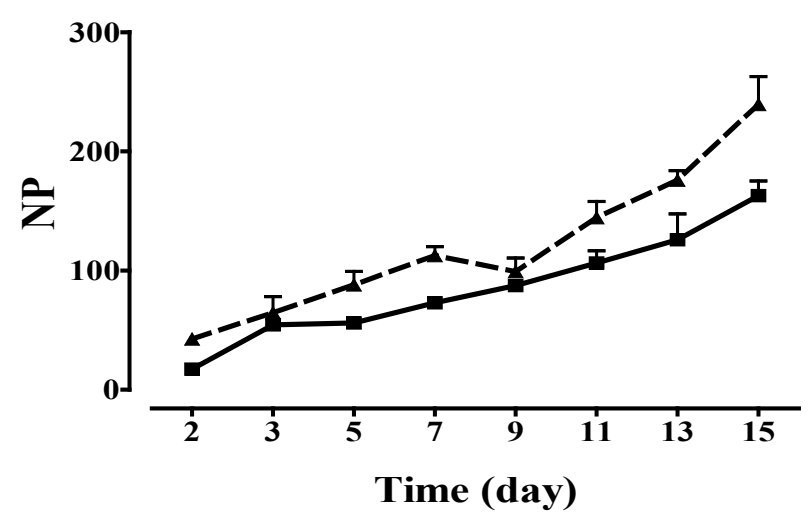

Figure 1: Number of $T$. vaginalis cultured in Diamond's Trypticase Yeast Maltose (TYM) media with $(\boldsymbol{\Delta})$ and without iron supplementation ( $\mathbf{m})$ during 15 days post inoculation. NP: Number of Parasites $(($ organism $/ \mathrm{ml}) \times 10000)$.
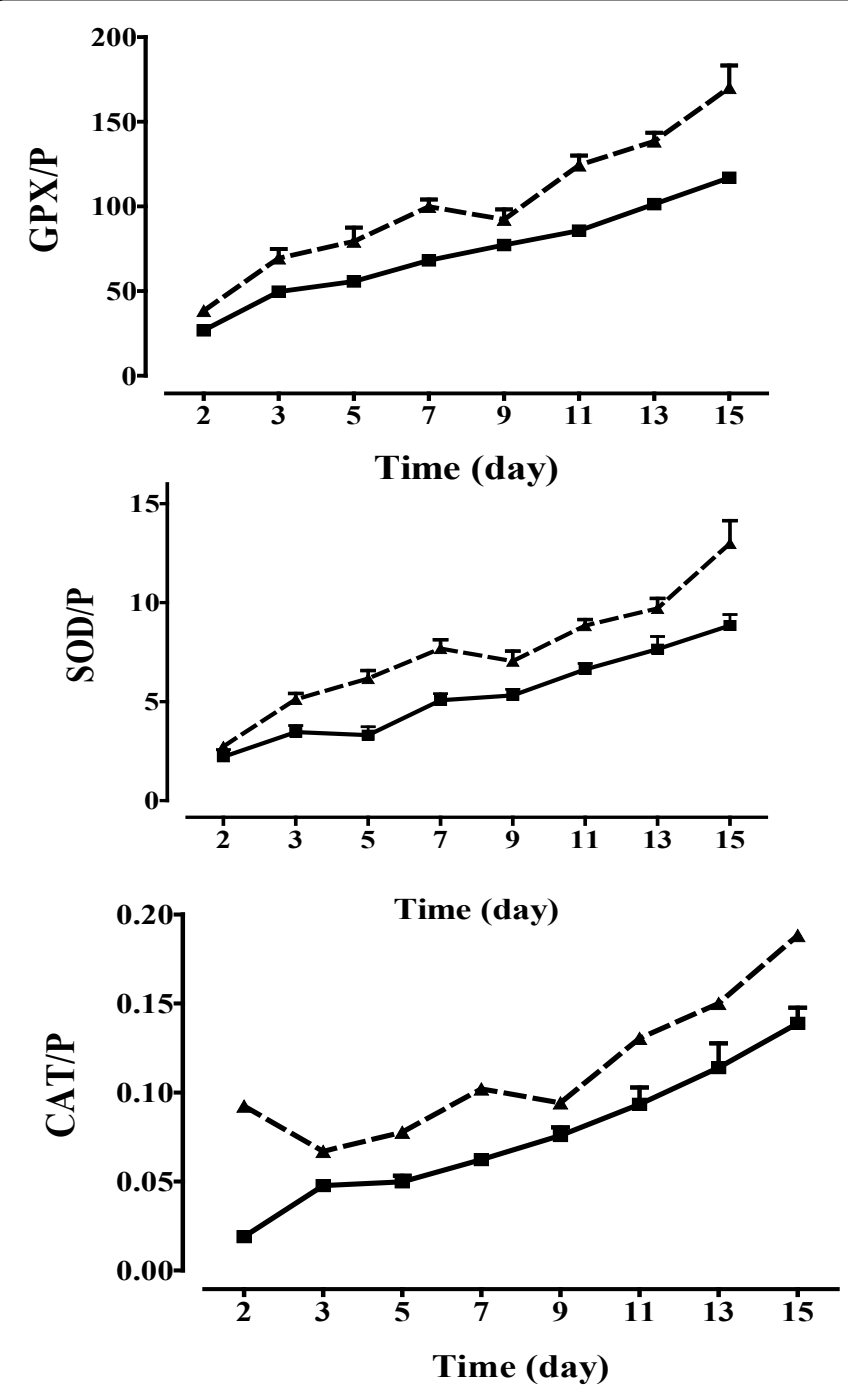

Figure 2: The activity of antioxidant enzymes (as unit/mg) in $T$. vaginalis cultured in Diamond's Trypticase Yeast Maltose (TYM) media with $(\boldsymbol{\Delta})$ and without iron supplementation ( $\mathbf{a}$ ) during 15 days post inoculation. Note that the average activity of each enzyme in the whole parasite population was divided into the parasite number. 
and 15 dpi; however, no significant difference was observed at 9 dpi.

A remarkable increase in GPx activity was evident in T. vaginalis cultured in iron-rich condition at 2, 3, 5, 7, 11, 13 and $15 \mathrm{dpi}$, but no significant difference was recorded at 9 dpi. Remarkable elevations were also observed in SOD activity in T. vaginalis grown in iron-rich medium at all samplings except for $2 \mathrm{dpi}$. The pattern of changes in the activity of Catalase (CAT) was different from that of GPx. According to our data, a significant increase in CAT activity was evident at $5,7,11$, 13 and 15 dpi but not at 2, 3 and 9 dpi.

\section{Discussion}

In the present study, the growth rate of $T$. vaginalis grown in the presence of iron was significantly higher compared to those cultured in medium with no iron supplementation. These observations reinforce the idea that increasing the level of iron favors the multiplication of the parasite, thus exacerbating the symptoms of the disease. Similarly, previous reports confirmed that $T$. vaginalis essentially requires iron for its growth in the human vagina, where the iron concentration is changing. This element could increase the parasite multiplication in different culture media [5,18]. It seems that iron is an important nutrient for the growth of different protozoan genera such as Entamoeba, Leishmania, Plasmodium and Trichomonads. Leishmania and Plasmodium can use haemoglobin as a source of iron by catabolism of the haem group [19]. Leishmania chagasi also expresses a NADPHdependent iron reductase capable of converting oxidized $\mathrm{Fe}^{3+}$ into the more soluble $\mathrm{Fe}^{2+}$ form [20]. In addition, the ability of Tritrichomonas foetus for the uptake of iron from the host environment may be a critical factor for its pathogenicity and virulence [21]. The parasite grows in the bovine vagina where lactoferrin is available and also invades the bovine uterus, where abundant transferrin exists [22].

Corroborating our data, Lehker and Alderete [18] assessed the responses of $T$. vaginalis to iron depletion or iron excess to evaluate the regulatory role of this element on certain properties of the parasite. They stated that, in comparison with organisms grown in excess iron, iron restriction resulted in greater than or equal to $80 \%$ lower rates of protein synthesis, greater than or equal to 3 -fold decreases in cell densities and 2.5-fold longer generation time.

The role of iron in T. vaginalis has been shown to be associated with the virulence of the parasite in mice [7]. In addition to the increased virulence, iron also enhances the level of adherence and the cytotoxicity of Trichomonads [7].

High metabolic rate of the rapidly growing and multiplying parasites will produce large amounts of toxic by-products in the cells. Excessive in vivo generation of these products can adversely affect the cell functioning. The accumulation of high concentrations of free radical, mainly the reactive oxygen species (ROS), could damage the cell phospholipid membranes and vital macromolecules [23]. This condition is considered as oxidative stress which is indicated by impairment of antioxidant systems [24]. The balance between free radicals and antioxidants may be disrupted in many diseases. Previous studies show that protozoan parasite infections such as Plasmodium sp. [25], Trypanosoma sp. [26], Trichomonas foetus [27] and some nematodes $[28,29]$ can induce oxidative stress in the host. However, defense mechanisms operating against reactive metabolites of molecular oxygen in T. vaginalis have not been studied in detail.

The results obtained here corroborate that iron has significant effect on the antioxidant activity of $T$. vaginalis in vitro. We revealed that the activities of GPx, SOD and CAT were significantly higher in iron-supplemented media. Higher numbers of parasites along with the increased amounts of antioxidant activities in the parasites grown in the presence of iron clearly indicate that this element enhances the strength of $T$. vaginalis to resist the vaginal defense mechanisms. Corroborating our data, Razavi et al. [30] studied the status of the activity of antioxidant enzymes in Trichomonas gallinae cultured in aerobic and anaerobic media. They revealed higher activities of GPx, SOD and CAT activities in Trichomonas cultured in both environments. These conditions stimulate the production of those protective enzymes at early and late stages of cultivation, depending upon the presence of oxygen in culture media. In contrast to these results, Ellis et al. [31] stated that $T$. vaginalis trophozoites lack the major peroxide-reducing enzymes, catalase and glutathione peroxidase. However, the presence of glutathione reductase and SOD activity suggested that some detoxification mechanisms may operate in T. vaginalis. This controversy is likely due to the sensitivity of $T$. vaginalis to oxygen above physiological levels which led to the lack of adequate peroxide reducing enzymes.

Antioxidant defense mechanisms have been indicated in other protozoans. Incubation of E. histolytica isolates under different culture conditions with an oxygen radical generating system induces an increase in SOD activity [32]. Therefore, it was suggested that regulation of SOD may support the parasite from invasion by oxygen metabolites. Although the specific activity of FeSOD in E. histolytica is reduced in the presence of a ferrous iron chelator (1,10-phenanthroline), the total activity was found to be increased substantially. It was hypothesised that at low $\mathrm{Fe}^{2+}$-levels in E. histolytica, the iron molecule in FeSOD may be replaced by an alternative divalent cation, like $\mathrm{Mn}^{2+}$. Also, it can be argued that the affinity of iron to the enzyme is much higher than to the repressor molecules.

In conclusion, it can be noted that iron-supplemented media stimulate not only the higher growth rates but also the higher activity of antioxidant enzymes (SOD, GPX and CAT) in different days post inoculation for $T$. vaginalis. These observations could suggest that positive regulations of antioxidant enzymes in the presence of iron may contribute to protect $T$. vaginalis from oxygen free radicals during tissue invasions.

\section{Acknowledgement}

The authors would like to thank the Research Council of Shiraz University and School of Veterinary Medicine, Shiraz University for financial and technical support of this study (Grant No.71-GR-VT-5).

\section{Conflict of Interest}

The authors declare no conflicts of interest.

\section{References}

1. Wölner-Hanssen P, Krieger JN, Stevens CE, Kiviat NB, Koutsky L, et al. (1989) Clinical manifestations of vaginal trichomoniasis. JAMA 261: 571-576.

2. Petrin D, Delgaty K, Bhatt R, Garber G (1998) Clinical and Microbiological Aspects of Trichomonas vaginalis. Clin Microbiol Rev 11: 300-317.

3. Heine P, McGregor JA (1993) Trichomonas vaginalis: a reemerging pathogen. Clin Obstet Gynecol 36: 137-144.

4. Diamond LS (1986) In vitro cultivation of the Trichomonadidae: a state of the art review. Acta Univ Carol Biol 30: 221-228.

5. Gorrell TE (1985) Effect of culture medium iron content on the biochemica composition and metabolism of Trichomonas vaginalis. J Bacteriol 161: 12281230.

6. Alderete JF, Provenzano D, Lehker W (1995) Iron mediates Trichomonas vaginalis resistance to complement lysis. Microb Pathog 19: 93-103.

7. Ryu JS, Choi HK, Min DY, Ha SE, Ahn MH (2001) Effect of iron on the virulence of Trichomonas vaginalis. J Parasitol 87: 457-460. 
Citation: Razavi SM, Foroudi E, Rakhshandehroo E, Nejati F, Nazifi S (2017) The Effects of Iron Supplementation on the Growth Rate and Antioxidant Activity of Trichomonas vaginalis. J Vet Sci Technol 8: 463. doi: 10.4172/2157-7579.1000463

8. Poli G (1993) Liver damage due to free radicals. Br Med Bull 49: 604-620.

9. Ramakrishna V, Jailkhani R (2007) Evaluation of oxidative stress in insulin dependent diabetes mellitus (IDDM) patients. Diagn Pathol 2: 22.

10. Schirmer RH, Schöllhammer T, Eisenbrand G, Krauth-Siegel RL (1987) Oxidative stress as a defense mechanism against parasitic infections. Free Radic Res Commun 3: 3-12.

11. Nazifi S, Razavi SM, Kianiamin P, Rakhshandehroo E (2011) Evaluation of erythrocyte antioxidant mechanisms: antioxidant enzymes, lipid peroxidation and serum trace elements associated with progressive anemia in ovine malignant theileriosis. Parasitol Res 109: 275-281.

12. Razavi SM, Nazifi S, Bateni M, Rakhshandehroo E (2011) Alterations of erythrocyte antioxidant mechanisms: Antioxidant enzymes, lipid peroxidation and serum trace elements associated with anemia in bovine tropical theileriosis. Vet Parasitol 180: 209-214.

13. Esmaeilnejad B, Tavassoli M, Asri-Rezaei S, Dalir-Naghadeh B, Malekinejad $\mathrm{H}$, et al. (2014) Evaluation of antioxidant status, oxidative stress and serum trace mineral levels associated with Babesia ovis parasitemia in sheep. Vet Parasitol 205: 38-45.

14. Linstead DJ, Bradley S (1988) The purification and properties of two soluble reduced nicotinamide: acceptor oxidoreductases from Trichomonas vaginalis. Mol Biochem Parasitol 27: 125-134.

15. Ellis JE, Cole D, Lloyd D (1992) Influence of oxygen on the fermentative metabolism of metronidazole-sensitive and resistant strains of Trichomonas vaginalis. Mol Biochem Parasitol 56: 79-88.

16. Diamond LS (1957) The establishment of various trichomonads of animals and man in axenic cultures. J Parasitol 43: 488-490.

17. Aebi $\mathrm{H}$ (1984) Catalase in vitro. Methods Enzymol 105: 121-126.

18. Lehker MW, Alderete JF (1992) Iron regulates growth of Trichomonas vaginalis and the expression of immunogenic trichomonad proteins. Mol Microbiol 6: $123-132$

19. Wilson ME, Britigan BE (1998) Iron acquisition by parasitic protozoa. Parasito Today 14: 348-353

20. Wilson ME, Lewis TS, Miller MA, McCormick ML, Britigan BE (2002) Leishmania chagasi: uptake of iron bound to lactoferrin or transferrin requires an iron reductase. Exp Parasitol 100: 196-207.
21. Kulda J, Poislova M, Suchan P, Tachezy J (1998) Enhancing effect of iron on experimental infection of mice by Tritrichomonas foetus. Parasitol Res 85 692-699.

22. Tachezy J, Kulda J, Bahníková I, Suchan P, Rázga J, et al. (1996) Tritrichomonas foetus: iron acquisition from lactoferrin and transferrin. Exp Parasitol 83: 216-228.

23. Wiseman $H$, Halliwell $B$ (1996) Damage to DNA by reactive oxygen and nitrogen species: role in inflammatory disease and progression to cancer. Biochem J 313: 17-29.

24. Hirotomo K, Horie T, Ishikawa S, Tsuda C, Kawakami S (2010) Oxidative stress in skeletal muscle causes severe disturbance of exercise activity without muscle atrophy. Free Radical Biol Med 48: 1252-1262.

25. Becker K, Tilley L, Vennerstrom JL, Roberts D, Rogerson S, et al. (2004) Oxidative stress in malaria parasite-infected erythrocytes: host-parasite interactions. Inter J Parasitol 34: 163-189.

26. Docampo R, Moreno SN (1984) Free radical metabolites in the mode of action of chemotherapeutic agents and phagocytic cells on Trypanosoma cruzi. Rev Infect Dis 6: 223-238.

27. Kitchener KR, Meshnick R, Fairfield A, Wang CC (1984) An iron-containing superoxide dismutase in Tritrichomoiias foetus. Mol Biochem Parasitol 12: 9599.

28. Jong EC, Mahmoud AA, Klebanoff SJ (1981) Peroxidase-mediated toxicity to schistosomula of Schistosoma mansoni. J Immunol 126: 468-471.

29. Kazura JW, Fanning MM, Blumer JL, Mahmoud AA (1981) Role of cell generated hydrogen peroxide in granulocyte-mediated killing of schistosomula of Schistosoma mansoni in vitro. J Clin Invest 67: 93-102.

30. Razavi SM, Changiz S, Rakhshandehroo E, Nejati F, Nazifi S (2016) An investigation on the pattern of antioxidant enzymes activities in Trichomonas gallinae cultured in aerobic and anaerobic conditions. Sci Parasitol 17: 36-42.

31. Ellis JE, Yarlett N, Cole D, Humphreys MJ, Lloyd D (1994) Antioxidant defences in the microaerophilic protozoan Trichomonas vaginalis: comparison of metronidazole-resistant and sensitive strains. Microbiol 140: 2489-2494

32. Bruchhaus I,Tannich E (1994) Induction of the iron-containing superoxide dismutase in Entamoeba histolytica by a superoxide anion-generating system or by iron chelation. Mol Biochem Parasitol 67: 281-288. 